\title{
Study on Reserve Capacity Optimization Model of Wind and Solar Power Generation System Based on Multi-Objective Optimization
}

\author{
Xiupeng Chen ${ }^{1}$, Feng $\mathrm{Lu}^{1}$, Yiping Cheng ${ }^{1}$ \\ ${ }^{1}$ North China Electric Power University, Beijing, China, 102206 \\ hunter2011@foxmail.com
}

Keywords: Reserve Capacity; Multi-Objective Optimization; Power Generation

\begin{abstract}
Rational optimization of the energy storage system can be configured to stabilize power fluctuations of wind and solar power generation systems. The use of batteries and complementary nature of ultra-capacitors, combined with real-time scheduling grid, we proposed a method to improve the overall economics of the energy storage system and supply reliability targets energy management strategies. Based on the energy management strategy, energy flow process is analyzed. The energy storage device function expressions are established based on an annual fee of full life-cycle theory and define power fluctuation rate expression, and finally simulation calculation is conducted by adopt immune genetic algorithm for multi-objective. It verifies the correctness and effectiveness of the optimization model and algorithm.
\end{abstract}

\section{Introduction}

Solar and wind power are the most widely distributed clean energy, but vulnerable to climate conditions, location and other environmental factors, showing a strong uncertainty [1]. Since both in time and have a certain geographical complementarity, while in storage, the inverter can share links, for cogeneration complementary resources, improve system reliability. Through the rational allocation of energy storage device capacity, can stabilize the output of wind and solar power generation system volatility, improve stability DC voltage of the inverter. Scholars at home and abroad off-grid wind and solar power generation system design, configuration and power energy storage device configuration optimization of capacity more, and for the study design and grid power system optimization and energy storage optimization has just started.

Energy storage technology makes the scenery more flexible energy management systems, but at present scenery storage battery as the energy storage element of common projects, large storage costs, limiting energy efficiency. So how to maximize the life of the battery pack is a major issue of new energy power generation system designer urgent need to address. In the current energy storage technology, the battery and super capacitor energy storage characteristics can achieve a good complement each other, and therefore such hybrid energy storage technology will be widely used in the landscape system [2]. In this paper, such hybrid energy storage system based on grid wind power generation energy storage capacity optimization configuration problems studied.

Paper [3] established a large-scale grid-connected solar hybrid power system dynamic analysis model is proposed based on the rate of change of power disturbances observed improved MPPT algorithm, but with the energy storage device. [4], it runs the index system of energy loss rate and miss rate as the energy storage capacity constrained optimal allocation model, did not carry out research on short-term power fluctuations. Paper [5] proposed an energy storage device based on the average power capacity configuration, optimization process does not take full account of the energy storage device characteristics.

\section{System Structure}

As a distributed power to the grid, scenery storage and grid hybrid power system includes wind farms, solar power plants and energy storage power. Figure 1, since the wind farms use different types of wind turbines, wind turbines and network mode are different, there is a direct and network, 
but also through the power electronic inverter and network, while the output of the PV array and energy storage systems are direct current, only transformed by the inverter to the Grid.

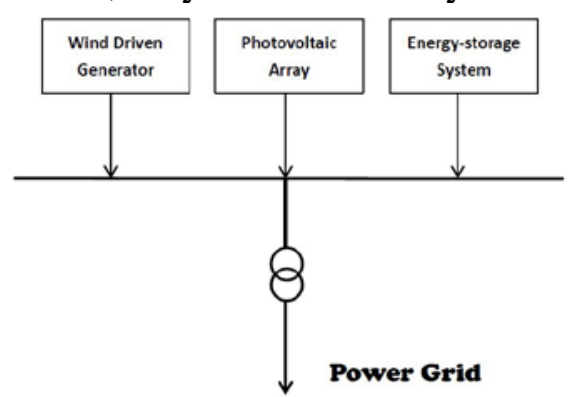

Fig. 1 Wind power generation system

\section{System Model}

Battery Model. Batteries commonly used lead-acid battery technology is the most mature, the most widely used. Lead-acid batteries are cheap, convenient universal energy than moderate, large capacity, good high temperature performance, high discharge efficiency. Battery equivalent circuit model processing [6], in Figure 2, by a controllable constant voltage source series resistor can dynamically reflect the characteristics of the battery charge and discharge, the equivalent circuit corresponding equation is:

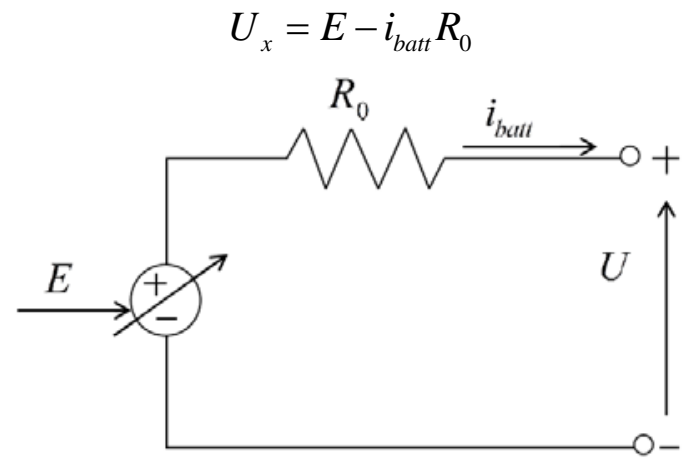

Fig. 2 Battery model

Where, $U_{x}$ for the battery terminal voltage, $E$ no-load voltage controllable voltage $i_{\text {batt }}$ source for battery current, $R_{0}$ the battery internal resistance.

If the battery's rated capacity $V_{x}(\mathrm{~A} \cdot \mathrm{h})$, a single battery for energy storage:

$$
E_{x \max }=V_{x} U_{x} / 10^{6}
$$

When the battery discharges, $D_{\text {odmax }}$ the maximum depth of discharge, the minimum storage capacity of a single battery:

$$
E_{x \min }=V_{x} U_{x}\left(1-U_{\text {od } \max }\right) / 10^{6}
$$

Battery discharge rate is usually the time, the battery output power rating:

$$
P_{x x}=V_{x} U_{x} / 10^{7}
$$

Ultra-capacitor Model. In addition, like super-capacitor battery with ordinary capacitor has higher energy density and power advantages, it also has to use a long time, charge and discharge rate, high charge-discharge efficiency and better high and low temperature performance. In addition, the material constituting the super capacitor toxicity tiny, little damage to the environment, and the use of super capacitors as the energy storage device does not require maintenance.

For the electric double layer super-capacitors consider the porous nature of the model is more complex, affecting the calculation process, in order to facilitate the calculation, usually an electric double layer capacitor using the simplified equivalent circuit model, the model consists of a simple RC equivalent circuit, The structure IS shown in Figure 3. 


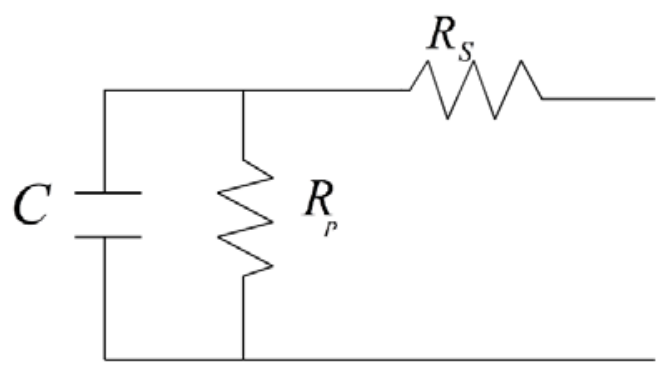

Fig. 3 Ultra-capacitor model

Super capacitor classical simplified model, the ideal capacitor $C$ with equivalent resistance $R_{b}$ are in parallel, and then with the equivalent impedance $R_{s}$ to the series. If each of the terminal voltage $U_{c}$ of super capacitor, capacitance value $V_{c}$, in actual operation, the terminal voltage of the super capacitor has a certain range. Written as $U_{c \min } \sim U_{c \max }$ the largest reserves of super capacitor is:

$$
\begin{aligned}
& E_{c \max }=0.5 V_{c} U_{c \max }^{2} /\left(3.6 \times 10^{9}\right) \\
& E_{c \text { min }}=0.5 V_{c} U_{c \text { min }}^{2} /\left(3.6 \times 10^{9}\right)
\end{aligned}
$$

Suppose the maximum operating current $I_{c \max }$ for the super-capacitor, the maximum output power of the super capacitor can be expressed as:

$$
P_{c \max }=U_{c \max } I_{c \max } / 10^{6}
$$

\section{Energy Management Strategy}

Scenery storage hybrid power system should meet the scheduling requirements of the grid, when the wind farms and photovoltaic power plants can not meet the schedule tasks, energy storage system should be able to replenish [7]. According to forecast power generation power plant, real-time access grid power dispatching value, resulting in supply and demand grid scheduling tasks and scenery generation difference:

$$
\Delta E=E_{l}-\left(E_{w}+E_{v}\right)
$$

Wherein; $E_{l}$ the power grid requirements, $E_{w}$ is wind power generation, $E_{v}$ photovoltaic power generation, $\Delta E$ the amount of power shortage.

At that time, $\Delta E>0$ instructions issued by the solar wind power to meet the scheduled task, the energy storage device can be charged.

At that time $\Delta E<0$, instructions issued by the solar wind power can not meet the scheduled task, the energy storage device to the power supply needs, the energy storage device is discharged.

Meanwhile, in order to prevent a sudden power loss, caused by power quality plunged, to the detriment of the entire power grid, energy storage devices must be able to quickly output power to support the system. Total power super capacitor and battery hybrid energy storage devices must be able to fill the power emitted by the loss of the grid, namely

$$
\begin{gathered}
P_{x n}+P_{c m} \geq \Delta P \\
\Delta P=P_{l}\left(t_{i}\right)-\left[P_{w}\left(t_{i}\right)+P_{v}\left(t_{i}\right)\right]
\end{gathered}
$$

Wherein; the power grid requirements for wind power generation, photovoltaic power generation, the amount of power shortage.

At that time, instructions issued by the solar wind power to meet the scheduled task, the energy storage device can be charged.

At that time, instructions issued by the solar wind power can not meet the scheduled task, the energy storage device to the power supply needs, the energy storage device is discharged.

$$
\begin{gathered}
P_{x n}+P_{c m} \geq \Delta P \\
\Delta P=P_{l}\left(t_{i}\right)-\left[P_{w}\left(t_{i}\right)+P_{v}\left(t_{i}\right)\right]
\end{gathered}
$$


Where: $\Delta P$ represents the instantaneous power grid dispatching missing, $P_{x n}$ indicates battery power issue, $P_{c m}$ indicated that the super capacitor emitted power.

In the energy storage device capable of ensuring a sufficient output power, the energy output should also be able to reach grid requirements grid scheduling tasks to meet the target, wind power photovoltaic sufficient in the case but also try to store the extra energy used to meet in the wind PV systems generate electricity when insufficient energy output. At this time, strike an energy storage device in storage

Save energy and meet the above requirements, such as the formula for the difference between the difference

$$
\Delta E^{\prime}=E_{c m}+E_{x n}-\Delta E
$$

In the formula, $\Delta E^{\prime}$ represents the amount of energy stored power grid power shortage, $E_{c m}$ shows super capacitor energy storage, and $E_{x n}$ represents the energy stored in the battery

At that time $\Delta E^{\prime}>0$, instructions stored in the energy storage device at the time $\Delta E^{\prime}<0$ of the power difference can fill the needs of the grid.

At that time, indicating that the power grid energy storage device is not enough to fill the demand. So, now we need to increase the capacity of energy storage systems in wind power, photovoltaic power generation when the rich as much as possible to store electrical energy when there is a lack of sufficient power generation output.

Taking into account the intermittent power supply volatility, combined with energy storage characteristics of the battery and super capacitor complementary, borne mainly by power generation and scheduling unequal power imbalance power generated frequent fluctuations in power; batteries not only take charge balance of power in the basic power, which can effectively prevent the battery charge and discharge frequently charge small cycles of discharge phenomena, and the battery charge and discharge are rated power, ensure that the battery is optimized working condition, extend battery life, increase energy storage system the overall economy.

\section{Optimization Model}

IEC 60300-3-3 (life cycle cost of the International Electrotechnical Commission to develop low computational standards) regulations in the life cycle cost of the equipment is from the planning, design, manufacture, installation, use, maintenance and disposal of the whole process the total charges accrued. This article will target energy storage system optimization model is defined as the sum of four costs, namely IEC standard acquisition costs, operating costs, maintenance costs, and disposal costs, and cited different scale factors indicate corresponding costs. The cost of the objective function are translated into an average annual cost of energy storage systems, conversion coefficient storage device using two annual depreciation value.

$$
\begin{aligned}
\min C & =C_{1}+C_{2}+C_{3}+C_{4} \\
& =\left(1+k_{o x x}+k_{m x x}+k_{d x x}\right) m f_{x} C_{x}+\left(1+k_{o x c}+k_{m x c}+k_{d x c}\right) n f_{c} C_{c}
\end{aligned}
$$

Where: $C_{1}$ represents the acquisition cost of the energy storage device, including the acquisition cost of the energy storage device and additional equipment. , Respectively, $C_{x}, C_{c}$ batteries and ultra-capacitors Unit; $m, n$ each represents the number of batteries and super capacitors; $f_{x}, f_{c}$ each represents annual depreciation value batteries and ultra-capacitors. The battery capacity is the extent of losses by depreciation process to represent the super capacitor depreciation calculated using the number of cycles.

$\mathrm{C}_{2}$ indicates the running cost of the energy storage device, comprising test, install, loss, outage costs, labor costs and other energy storage devices. Respectively, $k_{o x x}, k_{o x c}$ batteries and ultra-capacitors operational cost factor. 
$C_{3}$ represents the energy storage device maintenance costs, including maintenance costs fault detection costs money and fault. , Respectively, $k_{m x x}, k_{m x c}$ batteries and super capacitors maintenance cost factor.

$\mathrm{C}_{4}$ represents the energy storage device disposal costs, including the cost of scrap and salvage the energy storage device. , Respectively, $k_{d x x}, k_{d x c}$ batteries and ultra-capacitors disposal cost factor.

$$
\min f_{L D S P}=\frac{\sum_{i=1}^{N}\left|P_{l}\left(t_{i}\right)-\left[P_{w}\left(t_{i}\right)+P_{v}\left(t_{i}\right)+P_{x c}\left(t_{i}\right)\right]\right|}{\sum_{i=1}^{N} P_{l}(i)}
$$

Where: $P_{l}\left(t_{i}\right)$ indicates a stable power grid runtime;

$P_{w}\left(t_{i}\right)$ represents the actual wind turbine power generation;

$P_{v}\left(t_{i}\right)$ Photovoltaic power generation units represent actual;

$P_{x c}\left(t_{i}\right)$ represents the power generation energy storage device, including battery and ultra-capacitor power generation power generation $P_{c}\left(t_{i}\right)$.

$\mathrm{N}$ is the selected sampling interval points.

\section{Restrictions}

According to the energy management strategy and taking into account the grid power generation system operating characteristics of scenery, build to meet and network constraints scenery safe and reliable operation of power systems. needs.

Energy storage system should be able to replenish the amount of power can meet scheduling

$$
E_{x n}+E_{c m} \geq \Delta E_{\max }
$$

Total power supercapacitor battery and hybrid energy storage device must be able to fill the power emitted by the lack of power, namely:

$$
P_{x n}+P_{c m} \geq \Delta P
$$

This paper covers the time limit set unit is installed as its maximum capacity constraint. Number of batteries and super capacitors can be installed should be met:

$$
\begin{aligned}
& n \leq\left[\frac{S}{S_{x}}\right] \\
& m \leq\left[\frac{S}{S_{c}}\right]
\end{aligned}
$$

Where; $S$ is a given area;

$S_{x}$ Single area occupied for the battery;

$S_{c}$ Single super capacitor area occupied;

Since the battery energy density, we consider the battery as the energy provided under those conditions, no wind no light, the battery for at least comply with working hours $T$.

$$
n \geq \frac{T W_{l d}}{\eta V_{x} U_{x} D_{o d \max }}
$$

Where, $W_{l d}$ electricity demand per unit time; Respectively, $V_{x}, U_{x}$ monomer capacity and voltage of the battery. $D_{\text {od } \max }$ is the maximum depth of discharge of the battery, the battery 
discharge power $\eta$. To extend the service life of the energy storage means, energy storage device power operation within a certain range. Which is,

$$
\begin{gathered}
P_{x}\left(t_{i}\right)=P_{x x}, \quad P_{x}(t)=0 \\
P_{c}\left(t_{i}\right) \leq P_{c \max } \\
E_{c \min } \leq E_{c} \leq E_{c \max }
\end{gathered}
$$

\section{Algorithm}

Multi-objective optimization algorithm has broad utility in many aspects of power system scheduling, power system load flow analysis, network planning, capacity planning. This paper is multi-target capacity based on immune genetic algorithm optimization model. Analogy immune process, antibody affinity for the antigen antibody recognizes an antigen size show strength capacity, the greater the affinity, it indicates that the antibody for the antigen recognition ability is stronger, the weak turn fall. For multi-target antigen (multi-objective function) is concerned, some antibodies (Pareto) perhaps for a certain antigen is strong in terms of affinity, but for other anti-principle may be weak, so the introduction of the overall affinity and local affinity to describe the merits of the antibody. Combined with genetic algorithms, specific algorithm flowchart four as follows:

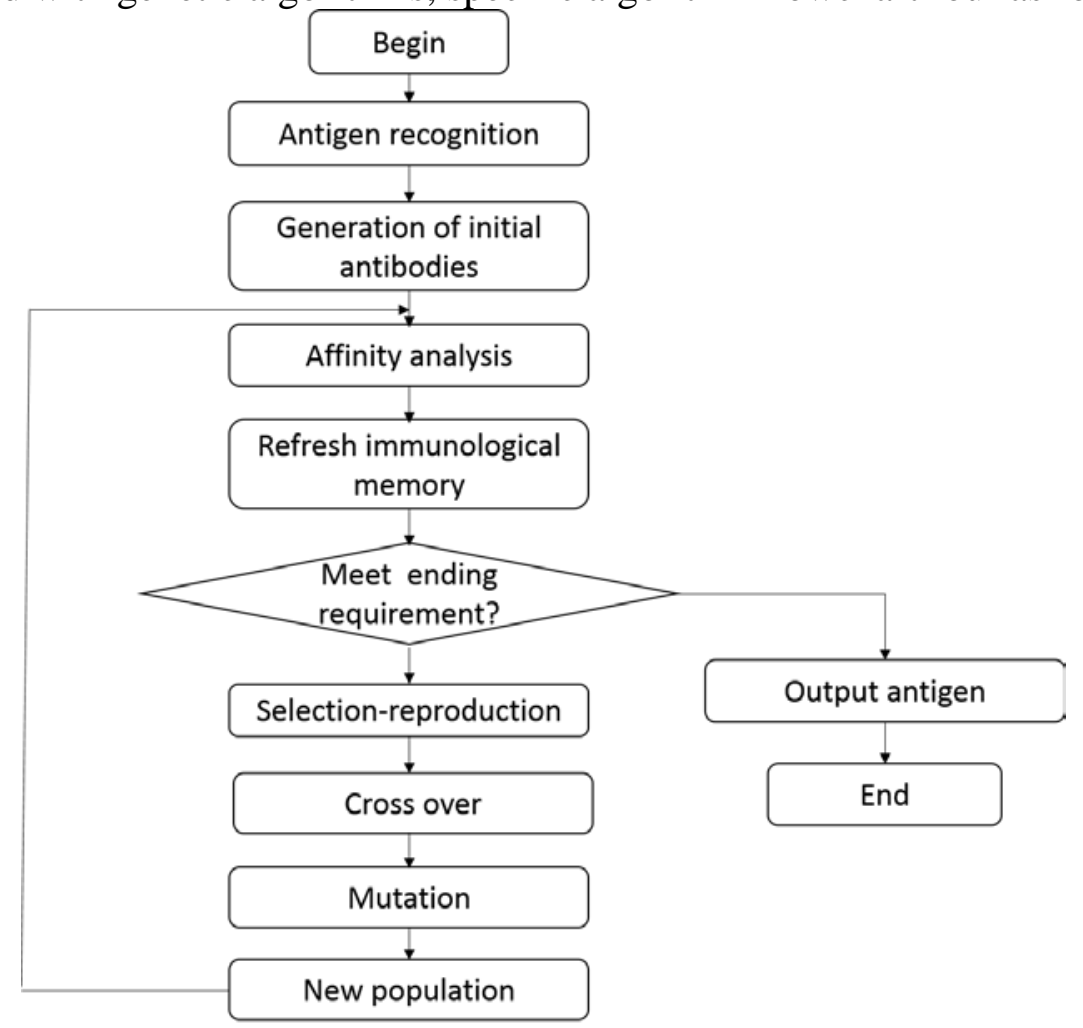

Scale wind power generation demonstration project of a wind power 60MW, PV 40MW, now make and network operation, and equipped with a storage capacity of the system. Build wind and solar power generation system model in MATLAB/SIMULINK, the energy storage device specific parameters in Table I: 
Table 1 Energy-storage device parameters

\begin{tabular}{|c|c|c|c|c|}
\hline \multicolumn{3}{|c|}{ Traditional lead accumulator } & \multicolumn{2}{|c|}{ Super capacitor } \\
\hline \multicolumn{2}{|c|}{ Energy-storage parameter } & Value & $\begin{array}{c}\text { Energy-storage } \\
\text { parameter }\end{array}$ & Value \\
\hline \multirow{2}{*}{$\begin{array}{c}\text { Rated } \\
\text { parameter }\end{array}$} & $\begin{array}{c}\text { Rated } \\
\text { capacity/A } • \mathrm{~h}\end{array}$ & 100 & Capacity/F & 3000 \\
\hline & Rated volt/V & 12 & Rated volt/V & 2.7 \\
\hline \multicolumn{2}{|c|}{ Discharge depth } & 0.4 & $\begin{array}{l}\text { Maximum working } \\
\text { current /A }\end{array}$ & 1500 \\
\hline \multicolumn{2}{|c|}{ Charge efficiency } & 0.75 & $\begin{array}{c}\text { Minimum working } \\
\text { volt/V }\end{array}$ & 0.8 \\
\hline \multicolumn{2}{|c|}{ Discharge efficiency } & 0.85 & $\begin{array}{c}\text { Charge-discharge } \\
\text { efficiency }\end{array}$ & 0.98 \\
\hline \multicolumn{2}{|c|}{ Cycle life/times } & 1000 & Cycle life/times & 500000 \\
\hline \multirow{3}{*}{ Cost } & $\begin{array}{l}\text { Maintenance cost } \\
\text { coefficient }\end{array}$ & 0.02 & $\begin{array}{l}\text { Maintenance cost } \\
\text { coefficient }\end{array}$ & 0.01 \\
\hline & $\begin{array}{l}\text { Operating cost } \\
\text { coefficient }\end{array}$ & 0.1 & $\begin{array}{c}\text { Operating cost } \\
\text { coefficient }\end{array}$ & 0.1 \\
\hline & $\begin{array}{c}\text { Disposal cost } \\
\text { coefficient }\end{array}$ & 0.08 & $\begin{array}{c}\text { Disposal cost } \\
\text { coefficient }\end{array}$ & 0.04 \\
\hline \multicolumn{2}{|c|}{ unit price /yuan } & 510 & unit price/yuan & 400 \\
\hline
\end{tabular}

Due to the power grid scheduling with real-time, apparently no longer storage is necessary and feasible, so choose one day wind and solar power plants within a hour of power as shown in Figure $\mathrm{V}$. purpose is to make the time period output power can be smoothly and meet scheduling requirements of the grid.

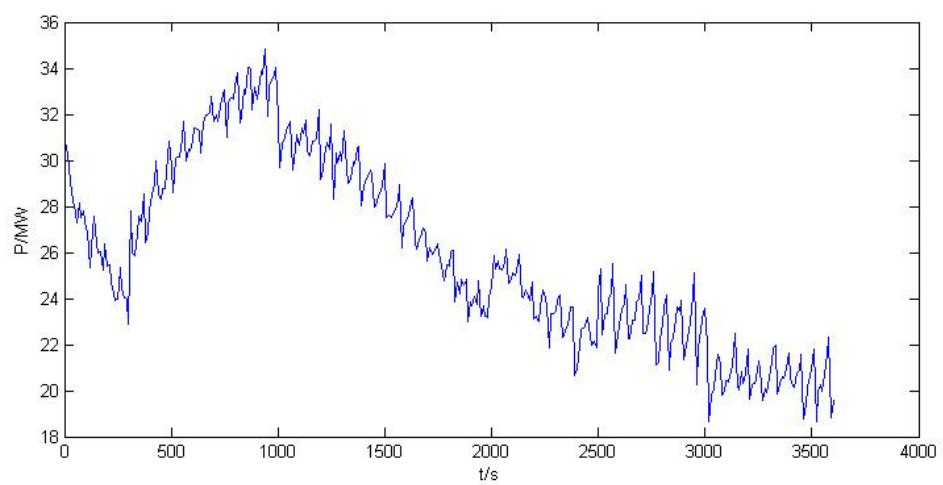

Fig. 6 Power plant output power

Figure 6 shows that power output has strong volatility, in order to smooth the output power, and to consider the output capacity of the energy storage device, multi-objective genetic optimization algorithm analysis and calculation, optimization calculation results are shown in Table 2. In order to compare the battery - economic superiority super-capacitor hybrid energy storage and battery single storage mode, respectively for the same set of data storage using a single storage mode and mixed mode calculation. 
Table 2 Optimumcalculation results

\begin{tabular}{|c|c|c|c|}
\hline $\begin{array}{c}\text { Energy-storage } \\
\text { method }\end{array}$ & $\begin{array}{c}\text { Number of } \\
\text { accumulators }\end{array}$ & $\begin{array}{c}\text { Number of super } \\
\text { accumulators }\end{array}$ & Total cost/yuan \\
\hline $\begin{array}{c}\text { Single energy } \\
\text { storage }\end{array}$ & 4580 & 0 & 2365846 \\
\hline $\begin{array}{c}\text { Hybrid energy } \\
\text { storage }\end{array}$ & 1252 & 204 & 720122 \\
\hline
\end{tabular}

Single use battery energy storage batteries 4580 requires an annual minimum fee of 2,365,846 yuan, while the use of hybrid energy storage battery 1252 only, super capacitor 204, the minimum annual cost of 720,122 yuan, can reduce system costs.

Figure 7 draw the power storage system to absorb compensation. Above the horizontal axis of the charging state, the horizontal axis following is a discharged state, through the storage configuration can effectively avoid the damage caused by power fluctuations.

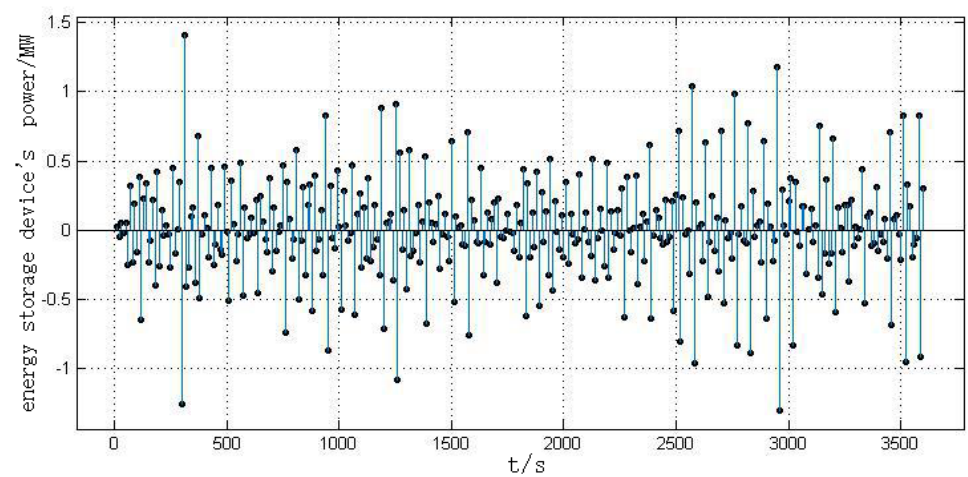

Fig. 7 Power energy storage system absorbs compensation

Shown in Figure 8, the battery load state curve gentler than many super capacitors, super capacitors reason to assume that the instantaneous power when starting complementary responsibilities, the role of continuous battery power, compared to non-super capacitor energy storage system as auxiliary equipment energy storage battery charge and discharge cycles increases, energy storage battery charge and discharge times are significantly reduced, effectively extending the service life of the storage battery, energy storage systems to reduce maintenance costs.

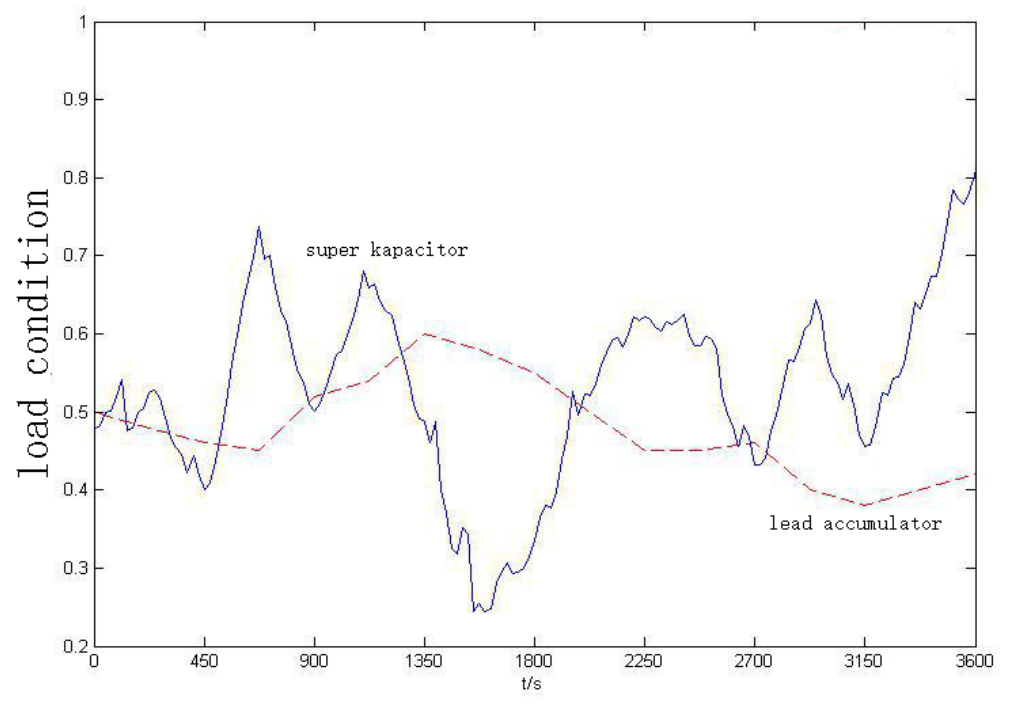

Fig. 8 load state energy storage devices 


\section{Conclusion}

This paper fully considered complementary operating characteristics of the battery and super-capacitors to reduce system operating costs and improve reliability as the goal, combined with the system energy management strategy, the optimal model to optimize the storage device capacity. Take full life-cycle cost theory as basis to conduct the establishment of storage capacity optimization objective function, in line with the actual situation. The objective function increase the volatility of power, the power level characterization intended effect, has practical significance. In this paper, immune genetic algorithm is used for multi-objective optimization, the results are accurate and reliable. The results show that the model is solved effectively play role of battery and super capacitor operating characteristics, stabilization effect is obvious and practical.

\section{References}

[1] KangLong Y, GuoHong X, Wu Jie, ChenSi Z. Several Research Review When Distributed Power And Access to Power System. Power System Technology, 2010,11: 43-47.

[2] WuHong B, Chen B. Wind and Solar Hybrid Power System Capacity Energy Storage Unit Optimization. Agricultural Engineering, 2011,04: 241-245.

[3] CaiGuo W, YangDe Y, etc. Large Scale Power System Modeling and Operational Characteristics Of. Wind Power System Technology, 2012,01: 65-71.

[4] Yang Jun, ZhangJian C, Gui X. Net Electricity Generation in Wind Energy Storage System Capacity Optimization Hybrid Configuration. Power System Technology, 2013,05: 1209-1216.

[5] ZhangMing R, LinCheng X, etc. A Grid Wind and Solar Power Generation Systems Modeling and Simulation. Grid And Clean Energy, 2014,01: 68-74, 80.

[6] YangGuo H, ZhuXiang F, Zhou X, etc. Optimal Allocation Based on Genetic Algorithm Wind Hybrid Energy Storage Capacity. Electric Drive, 2015,02: 50-53.

[7] HeYong Q, ZhangJian C, BaoXue N. Storage System Capacity Optimization. Grid Scenery Storage Hybrid Power System North China Electric Power University (Natural Science), 2012,04: $1-5$. 